\title{
USO DE MODELOS DEA EM AGRICULTURA: REVISÃO DA LITERATURA
}

\author{
Eliane Gonçalves Gomes ${ }^{1}$
}

Resumo: A rápida evolução da modelagem DEA, tanto em seus aspectos teóricos quanto em sua aplicação a casos de estudo reais, pode ser comprovada pela grande quantidade de artigos publicados. Uma das aplicações de destaque é na agricultura. Este artigo teve como objetivo fazer uma revisão da literatura sobre o uso de DEA na área agrícola. Foram encontradas 158 referências em periódicos internacionais e nacionais, cujas características são aqui descritas. Todas as referências estão listadas ao final do artigo.

Palavras-Chave: DEA; Agricultura; Estado da arte.

Abstract: DEA's modeling rapid evolution can be noticed by the great number of published papers, either in its theoretical basis or in its application to real case studies. One of these applications is in the agriculture field. This paper aimed at reviewing the state-ofthe-art of DEA modeling in agriculture. We found 158 references in both international and national journals. Their main characteristics are described here. All references are listed at the end of this paper.

Keywords: DEA; Agriculture; State-of-the-art.

\footnotetext{
${ }^{1}$ Empresa Brasileira de Pesquisa Agropecuária (Embrapa), e-mail: eliane.gomes@embrapa.br ENGEVISTA, v. 10, n. 1, p. 27-51, junho 2008
} 


\section{INTRODUÇÃO}

A publicação do modelo CCR por Abraham Charnes, William Cooper e Edwardo Rhodes (CHARNES et al., 1978) é reconhecida como o nascimento dos modelos de Análise de Envoltória de Dados (Data Envelopment Analysis DEA). Esta classe de modelos permite determinar a eficiência de uma unidade produtiva (Decision Making Unit DMU) comparativamente às demais, considerando-se os múltiplos recursos de que dispõe (inputs) e os múltiplos resultados alcançados (outputs). Desde então, a modelagem por DEA tornou-se popular na avaliação de eficiência, tanto no desenvolvimento de modelos teóricos quanto nas aplicações a casos reais. Isto é claramente constatado pelas inúmeras publicações científicas sobre DEA, listadas, por exemplo, nos artigos de Seiford (1997), Emrouznejad (2001), Tavares (2002), Phillips (2005). Esse universo é ainda expandido por relatórios técnicos (working papers), teses defendidas e artigos publicados em anais de eventos científicos.

Recentemente tiveram destaque números especiais de periódicos científicos sobre o tema, como por exemplo, o número 3 do volume 4 do International Journal of Information Technology \& Decision Making, de 2005; o número 10 do volume 55 do Journal of the Operational Research Society, de 2004; o número 2, do volume 22, ano de 2002, da revista Pesquisa Operacional. E, ainda, uma chamada de artigos no final de 2006 para uma edição especial sobre DEA no Journal of the Operational Research Society. São igualmente importantes os simpósios internacionais dedicados somente à modelagem DEA, como o International DEA Symposium, evento bianual, cuja $5^{\text {a }}$ edição ocorreu em Janeiro de 2007 e que terá sua $6^{\text {a }}$ edição, em Janeiro de 2009, sediada no Rio de Janeiro.

Lovell (2001) e Gattoufi et al. (2004a, 2004b) discutem as tendências da pesquisa em DEA, com indicações sobre sua relevância, difusão e aceitação em outras áreas da Pesquisa Operacional. Gattoufi et al. (2004a) destacam também os expressivos números do "universo" DEA: até agosto de 2001, a literatura de DEA contava com mais de 1.800 artigos, 1.100 deles publicados desde 1995. Os dados coletados por estes autores mostram que entre 1996 e 2001, 21\% dos artigos eram teóricos, $52 \%$ aplicados e $27 \%$ teóricos e aplicados. Estes números mostram a expressiva inserção desta classe de modelos na avaliação de eficiência em estudos de caso reais. Uma destas importantes áreas de aplicação é a área agrícola.

$\mathrm{Na}$ literatura foram encontrados alguns estudos sobre o estado da arte do uso de modelos de fronteiras de produção (paramétricas e não paramétricas; determinísticas e estocásticas) e avaliação de eficiência e produtividade em agricultura. Entretanto, não foram encontradas referências sobre revisão da literatura do uso específico de modelos DEA em agricultura. Dessa forma, este artigo tem como objetivo contribuir para esta classe de estudos e fazer uma revisão da literatura, tão extensa quanto possível, no que se refere ao uso de DEA em agricultura. $\mathrm{Na}$ maioria dos casos encontrados, a avaliação é de agricultores/fazendas e de regiões geográficas, sendo pontual em pesquisa agropecuária.

Este artigo está organizado como segue. No item 2 é feito um breve resumo dos modelos matemáticos clássicos da classe DEA. No item 3 apresenta-se o estado da arte no tema DEA e agricultura e mostram-se alguns números que refletem a potencialidade desta conjugação. É dado destaque ao seu uso com dados agrícolas brasileiros. No item 4 são feitas algumas considerações finais, seguidas do agradecimento. Finalmente, no item 6 listam-se todas as referências bibliográficas pesquisadas. 


\section{CONSIDERAÇÕES GERAIS SOBRE DEA}

DEA é tradicionalmente uma metodologia de análise de eficiência que usa Programação Linear para determinar uma fronteira de produção, linear por partes. O objetivo primário de DEA consiste em comparar um certo número de DMUs que realizam tarefas similares e se diferenciam nas quantidades de inputs que consomem e de outputs que produzem. Em modelagem por DEA são necessárias três etapas para a implementação do problema: definição e seleção de DMUs; seleção das variáveis (inputs e outputs); escolha e aplicação do modelo (tipo de retornos de escala; orientação; modelos avançados) (COOPER et al., 2000, 2004). A abordagem DEA pode ser dividida em duas etapas: determinação das medidas de eficiência e projeção na fronteira eficiente. A avaliação conjunta dos resultados dessas duas etapas pode ser de extrema utilidade na determinação de ações que melhorem o desempenho na área agrícola.

Há duas formulações (duais) equivalentes para DEA. De forma simplificada, pode-se dizer que uma das formulações (modelo do Envelope) define uma região viável de produção e trabalha com uma projeção de cada DMU na fronteira dessa região. A outra formulação (modelo dos Multiplicadores) trabalha com a razão de somas ponderadas de produtos e recursos, com a ponderação escolhida de forma mais favorável a cada DMU, respeitando-se determinadas condições. Os dois modelos DEA clássicos são o CCR e o BCC.

O modelo CCR (CHARNES et al., 1978) supõe retornos constantes de escala, ou seja, acréscimos nos recursos produzirão acréscimos proporcionais nos produtos. O modelo BCC (BANKER et al., 1984) considera situações de produção com variação de escala e não assume proporcionalidade entre inputs e outputs. Nesse modelo, o axioma da proporcionalidade é substituído pelo axioma da convexidade. De forma não matemática, no modelo BCC uma DMU é eficiente se, na escala em que opera, é a que melhor aproveita os recursos de que dispõe (relação outputs/inputs).

Diversos aperfeiçoamentos e desenvolvimentos teóricos aos modelos DEA clássicos vêm sendo realizados ao longo dos anos. Conforme destacado por Charnes et al. (1994), os novos desenvolvimentos em DEA surgem para contornar problemas que surgem na aplicação do método. Formulações e detalhamentos de modelos DEA clássicos e avançados podem ser vistos em Cooper et al. $(2000,2004)$.

\section{ESTADO DA ARTE: DEA EM AGRICULTURA}

Diversos autores dedicaram-se a rever o uso de modelos de fronteiras de produção na área agrícola. Battese (1992) conduziu um estudo sobre os diversos modelos de fronteiras de produção e explorou o estado da arte do uso de fronteiras de produção paramétricas em agricultura. Aplicações de métodos de fronteiras de produção à agricultura de países em desenvolvimento foram resumidas por Bravo-Ureta, Pinheiro (1993). Neste estudo, dos 37 artigos referenciados, um usava DEA. Coelli (1995), além de fazer uma revisão dos diversos modelos de fronteiras de produção e de medidas de eficiência, completou o estudo de Bravo-Ureta, Pinheiro (1993). Dos 38 artigos encontrados por ele sobre o tema fronteiras de produção e avaliação de eficiência, apenas três empregavam DEA.

Thiam et al. (2001) atualizaram a revisão da literatura de Bravo-Ureta, Pinheiro (1993) para os países em desenvolvimento, e dos 51 artigos referenciados, somente dois referiam-se ao uso de DEA. Bravo-Ureta et al. (2007) expandiram a revisão de Thiam et al. (2001) e conduziram pesquisa sobre modelos de fronteiras de produção em nível de fazenda. Dos 569 estudos 
encontrados, 87 usaram modelos nãoparamétricos tipo DEA (muitos usavam mais de uma técnica ou tipo de análise).

Revisões sobre o uso de modelos DEA aplicados à agricultura do Leste Europeu (República Tcheca, Hungria e Alemanha Oriental) podem ser vistos em Sarris et al. (1999). Nesta mesma linha, Gorton, Davidova (2004) fizeram uma revisão de estudos de eficiência sobre o Centro e Leste Europeus e dos 10 artigos encontrados, dois usavam DEA e outros dois combinavam DEA e outras técnicas de avaliação de eficiência.

Outras revisões da literatura também merecem destaque. $\mathrm{Na}$ revisão feita por Seiford (1997), de um total de 800 artigos listados entre 1978 e 1996, o autor encontrou dez referências ao uso de DEA na área agrícola. Sowlati (2005) fez uma revisão de estudos de eficiência na área agroflorestal. Picazo-Tadeo, ReigMartínez (2007) publicaram uma revisão sobre estudos de eficiência que consideram externalidades ambientais da agricultura. Sharma, Leung (2003) conduziram revisão de estudos com DEA e SFA (Fronteiras Estocásticas) especificamente em aquicultura (produção de camarão, carpa e tilápia). Alene et al. (2006) reviram estudos que comparam DEA e SFA em diversas áreas da agricultura.

A pesquisa resumida neste artigo foi feita entre março e julho de 2007. Foram pesquisadas as bases de dados Web of Science, Scopus, ProQuest, Compendex, WebSpiris, EbscoHost; WilsonWeb, Agricola, Agronbase, AgEcon Search, CIAT e Scielo. Algumas delas permitem acesso aos artigos completos, outras apenas ao resumo. Foram encontradas 158 referências ao uso de modelos DEA em agricultura em artigos publicados em periódicos científicos. 101 deles foram acessados como artigos completos e 57 como resumo. Alguns tratavam somente do uso de DEA, mas a maioria combinava diversos modelos de fronteiras de produção e estatísticos. Foi também feita uma pesquisa na base de Currículos
Lattes, do CNPq, para identificar publicações de autores nacionais. Estas representam, neste artigo, $11,5 \%$ do total encontrado (20 publicações), sendo duas em periódicos internacionais e o restante em periódicos nacionais.

Não foram aqui considerados os artigos publicados em anais de congressos, relatórios técnicos, dissertações de mestrado e teses de doutorado (estes dois últimos, exceto para o caso brasileiro). Foram somente registrados os artigos publicados em periódicos de circulação nacional e internacional, com júri formalizado, e que constavam das bases acima mencionadas (com exceção de alguns periódicos nacionais).

Os artigos foram descritos segundo: (1) tipo de tema ou produto, (2) localização geográfica do estudo de caso, (3) tipo e quantidade de DMUs, (4) ano dos dados, (5) tipo de artigo, (6) modelo DEA empregado, (7) modelos adicionais, (8) variáveis (inputs e outputs), (9) periódico, (10) ano de publicação. No caso dos resumos, as informações (1), (2), (3), (9) e (11) puderam ser descritas para todos; as demais somente puderam ser preenchidas para alguns. A Tabela 1 resume a classificação proposta e apresenta a freqüência relativa encontrada em cada caso.

Pela análise da Tabela 1 verificase que o tema mais freqüente é 'agricultura', seguido de 'agropecuária' e 'pecuária de leite'. O tema 'agropecuária' está mais ligado aos casos que avaliam eficiência de localizações geográficas; os demais referem-se, em sua maioria, à avaliação de produtores rurais ou fazendas.

Quanto à localização geográfica, não há uma tendência nas análises. Entretanto, ressalta-se que a freqüência relativa de artigos sobre estudos de caso brasileiros está majorada em relação ao total, pois foram consideradas todas as publicações encontradas, inclusive as de periódicos nacionais que não constavam das bases de dados mencionadas.

Os tipos de unidades de avaliação 
também variam, mas concentram-se na avaliação de produtores rurais/fazendas. A obtenção de dados nestes casos é geralmente feita via questionários de campo. Grande parte dos autores comenta sobre a dificuldade de obtenção de dados confiáveis neste tipo de pesquisa, o que interfere na qualidade dos mesmos e conduz ao descarte de uma grande quantidade de produtores (DMUs) durante a análise.

As quantidades de DMUs também variam de caso a caso. Para o caso de cooperativas, a média foi de 41 DMUs; mediana $=40 ;$ desvio padrão $=28$; máximo $=101 ;$ mínimo $=10$. Para o caso de divisões geográficas: média $=103$; mediana $=41$; desvio padrão $=175$; máximo $=754 ;$ mínimo $=8$. Para o caso de as DMUs serem fazendas/agricultores: média $=215 ;$ mediana $=117$; desvio padrão $=305 ;$ máximo $=1742 ;$ mínimo $=$ 8.

Os artigos encontrados são na grande maioria de artigos de aplicação prática de modelos DEA. Entretanto, há artigos com motivação teórica cujo exemplo é da área agrícola ou cuja aplicação necessitou de desenvolvimento teórico em DEA. Estes representam cerca de $14 \%$ do total.

Tabela 1: Resumo dos artigos encontrados.

\begin{tabular}{|c|c|c|}
\hline \multicolumn{2}{|c|}{$\begin{array}{l}\text { Tema ou produto } \\
\text { agropecuário }\end{array}$} & $\begin{array}{l}\text { Pesquisa Agropecuária (1,9\%); Horticultura (2,5\%); Pecuária de corte (exceto } \\
\text { suínos) }(3,1 \%) \text {; Agroflorestal }(3,8 \%) \text {; Suinocultura }(4,4 \%) ; \text { Aquicultura }(5,1 \%) ; \\
\text { Pecuária de leite (17,1\%); Agropecuária (produção animal) }(19,1 \%) ; \text { Agricultura } \\
\text { (produção vegetal - grãos, soja, milho, trigo, algodão, arroz, citrus, outros) } \\
(43,0 \%)\end{array}$ \\
\hline \multicolumn{2}{|c|}{ Localização geográfica } & $\begin{array}{l}\text { Costa Rica, Escócia, Filipinas, Indonésia, Mongólia, Nepal, Nova Zelândia, } \\
\text { Reino Unido, República Tcheca e Eslováquia, Tailândia, Itália, Ucrânia, Vietnã, } \\
\text { Bengali, Zimbabwe (0,6\% cada); Canadá, Coréia, Costa do Marfim, Finlândia, } \\
\text { Hawai, Hungria, Noruega, Paquistão, Polônia, Tanzânia (1,3\% cada); África do } \\
\text { Sul, Bangladesh, Dinamarca, Eslovênia, Etiópia, Japão, Oman, Rússia }(1,9 \% \\
\text { cada); Alemanha, Austrália, França, Índia, Taiwan, Diversos países em uma } \\
\text { mesma análise (2,5\% cada); China, Grécia (3,2\% cada); Holanda }(3,9 \%) ; \\
\text { Turquia (5,1\%); Espanha (7,0\%); Estados Unidos (12,9\%); Brasil }(12,7 \%) *\end{array}$ \\
\hline \multirow[t]{2}{*}{ DMUs } & Tipo & $\begin{array}{l}\text { Plantas de processamento, Regiões }(0,7 \% \text { cada); Condados, Distritos, } \\
\text { Embarcações, Parcelas, Províncias }(1,3 \% \text { cada); Centros de pesquisa }(2,0 \%) ; \\
\text { Estados, Municípios, Países }(2,7 \%) ; \text { Empresas agrícolas }(3,4 \%) ; \text { Cooperativas } \\
(6,8 \%) ; \text { Fazendas/produtores rurais }(78,6 \%)\end{array}$ \\
\hline & Quantidade & $\begin{array}{l}\text { Média }=180 ; \text { Mediana }=88 ; \text { Desvio padrão }=281 ; \text { Máximo }=1742 ; \text { Mínimo }= \\
8\end{array}$ \\
\hline \multicolumn{2}{|c|}{ Ano dos dados } & de 1980 até 2005 \\
\hline \multicolumn{2}{|c|}{ Tipo de artigo } & Teórico + Prático $(13,9 \%)$; Prático $(86,1 \%)$ \\
\hline \multicolumn{2}{|c|}{ Modelo DEA empregado } & $\begin{array}{l}\text { Aditivo }(0,5 \%) \text {; IRS-I ( } 0,9 \%) \text {; Variáveis ambientais }(0,9 \%) \text {; FDH }(1,4 \%) ; \\
\text { Variáveis exógenas }(1,4 \%) \text {; NIRS-I (2,8\%); Outputs indesejáveis }(3,8 \%) ; \\
\text { Variáveis não discricionárias }(4,3 \%) \text {; DEA Malmquist }(8,1 \%) ; \text { BCC-O }(9,5 \%) \text {; } \\
\text { CCR-O }(9,5 \%) \text {; Outros }(10,4 \%) * * \text { CCR-I }(20,0 \%) \text {; BCC-I }(26,5 \%)\end{array}$ \\
\hline \multicolumn{2}{|c|}{ Modelos adicionais } & $\begin{array}{l}\text { TFP }(8,1 \%) \text {, Testes estatísticos }(18,3 \%) \text {, Fronteiras estocásticas }(18,9 \%) \text {, } \\
\text { Modelos de regressão }(23,7 \%) \text {, Outros }(10,1 \%) \text {, Não usam modelos além de } \\
\text { DEA }(20,9 \%)\end{array}$ \\
\hline \multirow[t]{2}{*}{ Variáveis } & Inputs $* * *$ & $\begin{array}{l}\text { Herbicidas }(0,9 \%) \text {; Juros }(0,9 \%) \text {; Irrigação }(2,7 \%) \text {; Tamanho do rebanho } \\
(2,7 \%) \text {; Ração }(3,3 \%) \text {; Pesticidas }(3,6 \%) ; \text { Máquinas }(6,3 \%) \text {; Capital }(11,8 \%) ; \\
\text { Insumos variáveis }(12,1 \%) \text {; Fertilizantes }(13,6 \%) \text {; Área agrícola }(16,9 \%) \text {; Mão- } \\
\text { de-obra }(25,1 \%)\end{array}$ \\
\hline & Outputs $* * *$ & $\begin{array}{l}\text { Índices sintéticos (preços ou quantidade) }(2,9 \%) \text {; Vendas }(3,6 \%) \text {; Produtividade } \\
(7,2 \%) \text {; Valor da produção }(7,2 \%) \text {; Renda/rendimento/retorno financeiro } \\
(16,7 \%) \text {; Produção (vegetal e/ou animal) }(62,3 \%)\end{array}$ \\
\hline \multicolumn{2}{|l|}{ Periódico } & $\begin{array}{l}\text { Annals of Operations Research, Journal of Econometrics, Investigação } \\
\text { Operacional, Sustainable Development ( } 0,6 \% \text { cada); Aquaculture, Canadian } \\
\text { Journal of Agricultural Economics, European Journal of Operational Research, }\end{array}$ \\
\hline
\end{tabular}




\begin{tabular}{|l|l|}
\hline & Food Economics - Acta Agriculturae Scandinavica, Section C, Irrigation and \\
Drainage, Journal of Sustainable Agriculture, Journal of the Agricultural \\
Association of China, Pakistan Journal of Biological Sciences, Pesquisa \\
Operacional, Revista de Economia e Sociologia Rural, Transactions of the \\
Chinese Society of Agricultural Engineering (1,3\% cada); Applied Economics \\
Letters, Journal of Comparative Economics, Forest Policy and Economics, \\
Journal of Environmental Management, Omega, Quarterly Journal of \\
International Agriculture, Revista de Economia e Agronegócio (1,9\% cada); The \\
Australian Journal of Agriculture and Resource Economics (2,5\%); European \\
Review of Agricultural Economics, Journal of Agricultural Economics (3,2\% \\
cada); Journal of Productivity Analysis (4,4\%); Agricultural Systems (5,1\%); \\
Applied Economics (5,7\%); Agricultural Economics (9,5\%); outros (0,6\% cada)
\end{tabular}

* a freqüência relativa de artigos sobre estudos de caso brasileiros está majorada em relação ao total, pois foram aqui consideradas as publicações em periódicos nacionais que não constavam das bases de dados mencionadas; ** modelos DEA avançados (ex. IDEA, fronteira difusa, eficiência hiperbólica, restrições aos pesos etc.); *** foram listados apenas os mais citados; casos pontuais foram aqui descartados.

\section{Quanto aos modelos DEA} usados, a maioria dos artigos concentrouse nos modelos DEA clássicos, CCR e BCC, com orientação dependente de cada caso. É interessante notar que cerca de $10 \%$ dos artigos usaram modelos DEA avançados, o que mostra que ainda há potencialidades de novos desenvolvimentos e aplicações nesta área. Destaca-se uma parcela importante de artigos que usaram índices de Malmquist e DEA em suas análises. Estes referem-se sempre a estudos com dados de diversos períodos de tempo, no sentido de acompanhar a evolução do desempenho das unidades de avaliação do caso em questão. Ressalta-se também que cerca de $5 \%$ dos artigos trataram de outputs indesejáveis e variáveis de caráter ambiental, temas de ponta em modelagem DEA.

A maior parte dos artigos encontrados não fazem uso exclusivo de modelos DEA. Os autores usam modelos DEA e comparam seus resultados com os de outros modelos (no caso com modelos SFA e TFP, $18,9 \%$ e $8,1 \%$, respectivamente), usam os resultados de DEA como variável dependente de modelos de regressão, para identificar os fatores que afetam as medidas de eficiência $(23,7 \%)$, fazem uso de testes estatísticos $(18,3 \%)$, entre outros.

ENGEVISTA, v. 10, n. 1, p. 27-51, junho 2008
As variáveis usadas na modelagem DEA na área agrícola representam, na maioria dos casos, as relações clássicas de capital e trabalho. Como inputs, os mais referenciados foram mão-de-obra (familiar e/ou contratada), área usada na atividade agrícola, capital. Também receberam destaque o uso de insumos agrícolas diversos (fertilizantes, pesticidas, sementes, medicamentos, ração etc.) e máquinas e equipamentos. Como produto dos modelos DEA, geralmente foi usada a produção animal e/ou vegetal. Essas variáveis foram expressas em unidades físicas de medida ou em unidades monetárias.

Os artigos que usam DEA em agricultura foram na maioria publicados em periódicos da área agrícola, em especial na área de Economia Agrícola. As publicações em periódicos da área de Pesquisa Operacional representam cerca de $10 \%$ do total. Destacam-se nesta categoria Journal of Productivity Analysis, Omega, European Journal of Operational Research, Pesquisa Operacional, Annals of Operational Research e Investigação Operacional.

Quanto ao período de publicação, verifica-se que os últimos dez anos foram responsáveis por quase $92 \%$ das contribuições de DEA em agricultura. Ressalta-se que cerca de $26 \%$ das 
publicações foram entre 2006 e 2007, o que mostra os crescentes uso e interesse por DEA na área agrícola.

A Tabela 2 traz a relação dos artigos pesquisados, por tipo de produto ou tema. A busca por produto ou tema é uma das formas de consulta mais efetuada em pesquisas em bases de dados.

\subsection{USO NO CASO BRASILEIRO}

Conforme mencionado foram encontradas 20 publicações com aplicações a casos de estudo brasileiros. A Tabela 3 apresenta um resumo destes artigos. Destes, metade refere-se ao país, com análise da agropecuária dos estados brasileiros (PEREIRA et al., 2002; VICENTE, 2004) ou de algumas localizações do país, com estudos sobre centros de pesquisa agropecuária (SOUZA et al., 1999, 2007; SOUZA, 2006), fazendas de pecuária de leite (HOMEM DE SOUZA, ALVES, 2003), municípios (OTSUKI et al., 2002; BAPTISTA et al., 2004; SENA, PEREIRA FILHO, 2006), fazendas típicas (HELFAND, LEVINE, 2004).

Tabela 2: Artigos pesquisados por tipo de produto ou tema.

\begin{tabular}{|c|c|}
\hline Tema & Citação \\
\hline Agricultura & $\begin{array}{l}\text { Färe et al. (1985); Ray (1985); Fernandez-Cornejo (1994); Piesse et al. (1996); } \\
\text { Mao, Koo (1997); Chambers et al. (1998); Sueyoshi et al. (1998); Chang, Hsieh } \\
\text { (1998); Sedik et al. (1999); Sueyoshi (1999); Wadud, White (2000); Balmann, } \\
\text { Czasch (2001); Kim (2001); Silva, Sampaio (2002); Aldaz, Millán (2003); } \\
\text { Audibert et al. (2003); De Koeijer et al. (2003); Gorton et al. (2003); Peng (2003); } \\
\text { Lin et al. (2004); Nkamleu (2004); Rodriguez-Diaz et al. (2004a, 2004b); Sarker, } \\
\text { De (2004); Lissitsa, Odening (2005); Timmer, Los (2005); Dharmapala, Zaibet } \\
\text { (2006); Gocht, Balcombe (2006); Horie, Yamaguchi (2006); Osborne, Trueblood } \\
\text { (2006); Tipi, Rehber (2006); Zong (2006); Chen et al. (2007) }\end{array}$ \\
\hline Agroflorestas & $\begin{array}{l}\text { Kao (1993); Bogetoft et al. (2003); Lee (2005); Bosetti, Locatelli (2006); Diaz- } \\
\text { Balteiro et al. (2006) }\end{array}$ \\
\hline Agropecuária & $\begin{array}{l}\text { Haag et al. (1992); Chavas, Aliber (1993); Chavas, Cox (1994); Mathijs et al. } \\
\text { (1999); Rusielik, Swityk (1999); Thiele, Brodersen (1999); Brümmer (2001); } \\
\text { Zofio, Lovell (2001); Lansink et al. (2002); Otsuki et al. (2002); Pereira et al. } \\
\text { (2002); Baptista et al. (2004); Gomes, Mangabeira (2004); Helfand, Levine } \\
\text { (2004); Latruffe et al. (2004); Managi, Karemera (2004); Paul et al. (2004); Rao, } \\
\text { Coelli (2004); Vicente (2004); Coelli, Rao (2005); Gomes et al. (2005, 2006); } \\
\text { Sena, Pereira Filho (2006); Cherchye, Puyenbroeck (2007); Haji (2007) }\end{array}$ \\
\hline Algodão & Shafiq, Rehman (2000); Binici et al. (2006); Wossink, Denaux (2006) \\
\hline Aquicultura & $\begin{array}{l}\text { Sharma et al. (1999a); Felthoven (2002); Pascoe, Herrero (2004); Tingley, Pascoe } \\
\text { (2005); Sousa Júnior et al. (2005); Bozoglu et al. (2006); Cinemre et al. (2006); } \\
\text { Kaliba et al. (2007) }\end{array}$ \\
\hline Arroz & $\begin{array}{l}\text { Coelli et al. (2002); Nghiem, Coelli (2002); Dhungana et al. (2004); Krasachat } \\
\text { (2004); Kwon, Lee (2004); Lutfi et al. (2006) }\end{array}$ \\
\hline Café & Mosheim (2002); Binam et al. (2003) \\
\hline Cana-de-açúcar & De Koeijer et al. (2002) \\
\hline Citrus & $\begin{array}{l}\text { Reig-Martínez, Picazo-Tadeo (2004); Picazo-Tadeo, Reig-Martinez (2006a, } \\
\text { 2006b, 2007) }\end{array}$ \\
\hline Desenvolvimento rural & Chan, Chan (2000); Vennesland (2005) \\
\hline Grãos & Byrnes et al. (1987); Färe et al. (1997); Piesse et al. (2005) \\
\hline Horticultura & $\begin{array}{l}\text { Zaibet, Dharmapala (1999); Garcia (2005); Haji, Andersson (2006); Lansink, } \\
\text { Bezlepkin (2006) }\end{array}$ \\
\hline Pecuária de corte & $\begin{array}{l}\text { Gillespie et al. (1997); Fraser, Hone (2001); Zaibet et al. (2004); Abreu et al. } \\
\text { (2006); Kleinhanss et al. (2007) }\end{array}$ \\
\hline Pecuária de leite & $\begin{array}{l}\text { Ferrier, Porter (1991); Cloutier, Rowley (1993); Lund et al. (1993); Yang et al. } \\
\text { (1994); Manos, Psychoudakis (1997); Turk (1998); Fraser, Cordina (1999); } \\
\text { Jaforullah, Whiteman (1999); Psychoudakis, Dimitriadou (1999); Reinhard et al. }\end{array}$ \\
\hline
\end{tabular}




\begin{tabular}{|l|l|}
\hline & $\begin{array}{l}\text { (2000); Singh et al. (2001); Tupy, Yamaguchi (2002); Homem de Souza, Alves } \\
\text { (2003); Mbaga et al. (2003); Tupy et al. (2003a, 2003b); Alvarez, Arias (2004); } \\
\text { Kaliba (2004); Kuosmanen et al. (2004); Santos et al. (2004); Chaaban et al. } \\
\text { (2005); Fraser, Graham (2005); Hansen, Stokstad (2005); Balcombe et al. (2006); } \\
\text { Barnes (2006); Stokes et al. (2007) }\end{array}$ \\
\hline Pesquisa agropecuária & Souza et al. (1999, 2007); Souza (2006) \\
\hline Suinocultura & $\begin{array}{l}\text { Piot-Lepetit, Rainelli (1996); Sharma et al. (1997, 1999b); Piot-Lepetit, Vermersch } \\
\text { (1998); Lansink, Reinhard (2004); Asmild, Hougaard (2006); Galanopoulosa et al. } \\
\text { (2006) }\end{array}$ \\
\hline Tabaco & Abay et al. (2004); Ören, Alemdar (2006); Sauer, Abdallah (2007) \\
\hline Trigo & $\begin{array}{l}\text { Jha et al. (2000); Alemdar, Ören (2006); Kamruzzaman et al. (2006); Malana, } \\
\text { Malano (2006) }\end{array}$ \\
\hline $\begin{array}{l}\text { Outros ou mais de um } \\
\text { produto agropecuário }\end{array}$ & $\begin{array}{l}\text { Thompson et al. (1990); Whittaker (1994); Somwaru, Nehring (1996); Piot-Lepetit } \\
\text { et al. (1997); Townsend et al. (1998); Jaenicke (2000); Bayarsaihan, Coelli (2003); } \\
\text { Chiremba, Masters (2003); Lansink, Silva (2004); Dimara et al. (2005); } \\
\text { Grazhdaninova, Lerman (2005); Alene et al. (2006); Gül (2006); Varga (2006) }\end{array}$ \\
\hline
\end{tabular}

Cinco artigos versavam sobre estudos no estado de São Paulo, quatro sobre fazendas, com análise da agropecuária (GOMES, MANGABEIRA, 2004; GOMES et al., 2005, 2006) e da pecuária de leite (TUPY et al., 2003b), um sobre cooperativas de leite (TUPY et al., 2003a). Os demais estudos referiramse à avaliação de eficiência de agricultores que praticavam pecuária de leite no estado de Minas Gerais (TUPY, YAMAGUCHI, 2002; SANTOS et al., 2004), pecuária de corte no Mato Grosso do Sul (ABREU et al., 2006), cultura do camarão no estado do Ceará (SOUSA JÚNIOR et al., 2005), de fazendas irrigadas em Pernambuco (SILVA, SAMPAIO, 2002).

Neste item, julgou-se importante citar as teses de doutorado e dissertações de mestrado nacionais que versaram sobre aplicações a dados brasileiros. Para tal, foram consultados o banco de teses da CAPES (que reúne os resumos dos trabalhos defendidos entre 1987 e 2006) e a plataforma de currículos Lattes. No banco de teses da CAPES foram encontradas cerca de 150 teses e dissertações que fizeram uso de modelos DEA em seu desenvolvimento. Destas,
25 empregaram-nos a estudos agrícolas brasileiros. As referência encontradas na plataforma Lattes já estavam contabilizadas na fonte anterior.

Os resumos das teses e dissertações encontrados mostraram que estas foram apresentadas entre $1998 \mathrm{e}$ 2006. Versaram sobre os seguintes temas: aquicultura, arroz, cooperativas de crédito rural, pecuária de corte (1 trabalho cada); agricultura (6); agropecuária (7); pecuária de leite (8). Os trabalhos cubriram diferentes extensões e localizações geográficas, como os estados da Bahia, Mato Grosso do Sul e região Nordeste (1 trabalho cada); Ceará, Pernambuco, Rondônia, São Paulo (2); Santa Catarina (3); Minas Gerais, Paraná e Brasil como um todo (4). Notou-se ainda uma diversidade em relação ao tipo de DMU considerado: assentamentos rurais, cooperativas de crédito rural, cooperativas e sociedades de capital, empresas agrícolas, estados, vilas rurais (1 trabalho cada); municípios (2); microrregiões (3); fazendas/produtores rurais (11). A Tabela 4 relaciona estas características a cada referência pesquisada. 
Tabela 3: Resumo dos artigos publicados para o caso brasileiro.

\begin{tabular}{|c|c|c|c|c|c|c|c|c|c|c|}
\hline Autor & $\begin{array}{l}\text { Abreu et al., } \\
2006\end{array}$ & $\begin{array}{c}\text { Baptista et al., } \\
2004\end{array}$ & $\begin{array}{c}\text { Gomes, } \\
\text { Mangabeira, } \\
2004\end{array}$ & $\begin{array}{l}\text { Gomes et al., } \\
2005\end{array}$ & $\begin{array}{l}\text { Gomes et al., } \\
2006\end{array}$ & $\begin{array}{c}\text { Helfand, } \\
\text { Levine, } 2004\end{array}$ & $\begin{array}{c}\text { Homem de Souza, } \\
\text { Alves, } 2003\end{array}$ & $\begin{array}{l}\text { Otsuki et al., } \\
2002\end{array}$ & $\begin{array}{l}\text { Pereira et al., } \\
2002\end{array}$ & $\begin{array}{l}\text { Santos et al., } \\
2004\end{array}$ \\
\hline Produto & $\begin{array}{l}\text { Pecuária de } \\
\text { corte }\end{array}$ & Agropecuária & Agropecuária & Agropecuária & Agropecuária & Agropecuária & Pecuária de leite & Agropecuária & Agropecuária & $\begin{array}{c}\text { Pecuária de } \\
\text { leite }\end{array}$ \\
\hline $\begin{array}{c}\text { Localização } \\
\text { Geográfica }\end{array}$ & $\begin{array}{l}\text { Mato Grosso } \\
\text { do Sul }\end{array}$ & Minas Gerais & São Paulo & São Paulo & São Paulo & Centro-Oeste & Brasil & Amazônia & Brasil & Minas Gerais \\
\hline \multirow[t]{2}{*}{ DMUs } & Fazenda & Municípios & Fazendas & Fazendas & Fazendas & $\begin{array}{l}\text { Fazendas } \\
\text { típicas }\end{array}$ & Fazendas & Municípios & Estados & Fazendas \\
\hline & 8 & 754 & 71 & 71 & 71 & 15 & 143 & 255 & 25 & $17 / 11$ \\
\hline Ano & $1995-2002$ & 1996 & 2001 & 2001 & 2001 & 1995-1996 & 2002 & 1995 & 1970-1996 & 1999-2003 \\
\hline Modelo & CCR-I; BCC-I & $\mathrm{FDH}$ & BCC-I & $\begin{array}{c}\text { BCC-I; } \\
\text { camadas de } \\
\text { isoeficiência }\end{array}$ & $\begin{array}{c}\text { BCC-I; } \\
\text { fronteira difusa }\end{array}$ & CCR-I & CCR-O; BCC-O & BCC-O & $\begin{array}{c}\text { CCR-O; DEA } \\
\text { Malmquist }\end{array}$ & CCR-I; BCC-I \\
\hline Inputs & $\begin{array}{l}\text { Despesas com } \\
\text { insumos, mão- } \\
\text { de-obra, } \\
\text { investimentos, } \\
\text { comissões, } \\
\text { viagens }\end{array}$ & $\begin{array}{c}\text { Área; mão-de- } \\
\text { obra; capital }\end{array}$ & $\begin{array}{l}\text { Área cultivada; } \\
\text { mão-de-obra; } \\
\text { uso de } \\
\text { máquinas }\end{array}$ & $\begin{array}{l}\text { Área cultivada; } \\
\text { mão-de-obra; } \\
\text { uso de } \\
\text { máquinas }\end{array}$ & $\begin{array}{c}\text { Área cultivada; } \\
\text { mão-de-obra; } \\
\text { uso de } \\
\text { máquinas }\end{array}$ & $\begin{array}{l}\text { Área; mão-de- } \\
\text { obra; tratores; } \\
\text { animais; } \\
\text { insumos }\end{array}$ & $\begin{array}{c}\text { Valor agregado } \\
\text { da terra, animais, } \\
\text { depreciação de } \\
\text { máquinas, } \\
\text { equipamentos, } \\
\text { benfeitorias; mão- } \\
\text { de-obra; gastos } \\
\text { com insumos; } \\
\text { outros gastos }\end{array}$ & $\begin{array}{c}\text { Área cultivada } \\
\text { mão-de-obra; } \\
\text { capital; }\end{array}$ & $\begin{array}{c}\text { Área; mão-de- } \\
\text { obra; } \\
\text { máquinas; } \\
\text { outros insumos }\end{array}$ & $\begin{array}{l}\text { Quantidade } \\
\text { anual de leite } \\
\text { produzida; } \\
\text { total de vacas; } \\
\text { área dedicada à } \\
\text { produção de } \\
\text { leite; custo } \\
\text { operacional } \\
\text { efetivo }\end{array}$ \\
\hline Outputs & $\begin{array}{l}\text { Vendas de } \\
\text { animais }\end{array}$ & $\begin{array}{l}\text { Produção } \\
\text { vegetal; } \\
\text { produção } \\
\text { animal }\end{array}$ & Renda líquida & Renda líquida & Renda líquida & $\begin{array}{c}\text { Valor bruto da } \\
\text { produção }\end{array}$ & Renda bruta & $\begin{array}{l}\text { Produção } \\
\text { animal; } \\
\text { produção } \\
\text { vegetal (arroz; } \\
\text { milho; feijão; } \\
\text { mandioca; } \\
\text { banana; } \\
\text { madeira) }\end{array}$ & $\begin{array}{l}\text { Produção } \\
\text { animal; } \\
\text { produção } \\
\text { vegetal }\end{array}$ & Margem bruta \\
\hline $\begin{array}{c}\text { Tipo de } \\
\text { artigo } \\
\end{array}$ & Aplicação & Aplicação & Aplicação & Aplicação & $\begin{array}{c}\text { Teoria + } \\
\text { Aplicação }\end{array}$ & Aplicação & Aplicação & Aplicação & Aplicação & Aplicação \\
\hline $\begin{array}{c}\text { Outros } \\
\text { modelos }\end{array}$ & $\begin{array}{c}\text { Componentes } \\
\text { principais }\end{array}$ & SFA & - & - & $\begin{array}{c}\text { Fronteira DEA } \\
\text { difusa }\end{array}$ & Regressão & $\begin{array}{c}\text { SFA; Método de } \\
\text { Varian }\end{array}$ & Regressão & - & - \\
\hline
\end{tabular}


Tabela 3: Resumo dos artigos publicados para o caso brasileiro (continuação).

\begin{tabular}{|c|c|c|c|c|c|c|c|c|c|c|}
\hline \\
\hline Autor & $\begin{array}{l}\text { Sena, Pereira } \\
\text { Filho, } 2006\end{array}$ & $\begin{array}{c}\text { Silva, } \\
\text { Sampaio, } 2002\end{array}$ & $\begin{array}{c}\text { Souza et al., } \\
1999\end{array}$ & $\begin{array}{c}\text { Souza et al., } \\
2007\end{array}$ & $\begin{array}{c}\text { Souza Júnior et } \\
\text { al., } 2005 * *\end{array}$ & Souza, 2006 & $\begin{array}{l}\text { Tupy, } \\
\text { Yamaguchi, } \\
2002\end{array}$ & $\begin{array}{c}\text { Tupy et al., } \\
2003 \mathrm{a}\end{array}$ & $\begin{array}{l}\text { Tupy et al., } \\
2003 \mathrm{~b}\end{array}$ & $\begin{array}{c}\text { Vicente, } 2004 \\
* *\end{array}$ \\
\hline Produto & Agropecuária & Agricultura & $\begin{array}{c}\text { Pesquisa } \\
\text { Agropecuária }\end{array}$ & $\begin{array}{c}\text { Pesquisa } \\
\text { Agropecuária }\end{array}$ & $\begin{array}{l}\text { Aqüicultura } \\
\text { (camarão) }\end{array}$ & $\begin{array}{c}\text { Pesquisa } \\
\text { Agropecuária }\end{array}$ & $\begin{array}{c}\text { Pecuária de } \\
\text { leite }\end{array}$ & $\begin{array}{c}\text { Pecuária de } \\
\text { leite }\end{array}$ & $\begin{array}{c}\text { Pecuária de } \\
\text { leite }\end{array}$ & Agropecuária \\
\hline $\begin{array}{c}\text { Localização } \\
\text { Geográfica }\end{array}$ & Bahia & Pernambuco & Brasil & Brasil & Ceará & Brasil & Minas Gerais & São Paulo & São Paulo & Brasil \\
\hline \multirow[t]{2}{*}{ DMUs } & Municípios & Colonos & $\begin{array}{l}\text { Centros de } \\
\text { pesquisa }\end{array}$ & $\begin{array}{l}\text { Centros de } \\
\text { pesquisa }\end{array}$ & Fazendas & $\begin{array}{l}\text { Centros de } \\
\text { pesquisa }\end{array}$ & Fazendas & Cooperativas & Fazendas & Estados \\
\hline & 146 & 195 & 34 & 37 & - & 37 & 54 & 10 & 30 & 27 \\
\hline Ano & $1995-1996$ & 1999 & - & $2001-2003$ & - & 2000 & 1998-1999 & 1999 & 2000 & 1970-1995 \\
\hline Modelo & $\begin{array}{c}\text { CCR-O; BCC- } \\
\text { O }\end{array}$ & $\begin{array}{c}\text { CCR-O; BCC- } \\
\text { O; FDH }\end{array}$ & CCR-I; NIRS-I & $\begin{array}{l}\text { BCC-I; índice } \\
\text { heurístico de } \\
\text { eficiência }\end{array}$ & - & BCC-I & CCR-I; BCC-I & CCR-I; BCC-I & BCC-I & $\begin{array}{c}\text { DEA } \\
\text { Malmquist; } \\
\text { orientação a } \\
\text { outputs }\end{array}$ \\
\hline Inputs & $\begin{array}{c}\text { Despesas com } \\
\text { salários, } \\
\text { insumo, outros }\end{array}$ & \begin{tabular}{|c|} 
Área irrigada; \\
gastos com \\
insumos; \\
capital; \\
irrigação; mão- \\
de-obra \\
\end{tabular} & $\begin{array}{c}\text { Custos de } \\
\text { pessoal; custos } \\
\text { operacionais; } \\
\text { custos de } \\
\text { capital }\end{array}$ & $\begin{array}{l}\text { Itens de } \\
\text { produção } \\
\text { técnico } \\
\text { científica, } \\
\text { tecnológica }\end{array}$ & - & $\begin{array}{l}\text { Custos de } \\
\text { pessoal; custos } \\
\text { operacionais; } \\
\text { custos de } \\
\text { capital }\end{array}$ & $\begin{array}{l}\text { Mão-de-obra; } \\
\text { gastos com } \\
\text { insumos; } \\
\text { capital } \\
\text { aplicado }\end{array}$ & $\begin{array}{c}\text { Ativo total; } \text { n. }^{\circ} \\
\text { de horas } \\
\text { trabalhadas }\end{array}$ & $\begin{array}{c}\text { Custo; mão-de- } \\
\text { obra; } \\
\text { quantidade de } \\
\text { concentrado }\end{array}$ & - \\
\hline Outputs & $\begin{array}{c}\text { Valor da } \\
\text { produção } \\
\text { agropecuária }\end{array}$ & $\begin{array}{c}\text { Valor da } \\
\text { produção de } \\
\text { culturas } \\
\text { irrigadas }\end{array}$ & $\begin{array}{l}\text { Produção } \\
\text { cientifica } \\
\text { agregada }\end{array}$ & Custo total & - & $\begin{array}{l}\text { Produção } \\
\text { cientifica } \\
\text { agregada }\end{array}$ & Renda bruta & Receita bruta & $\begin{array}{l}\text { Produção de } \\
\text { leite }\end{array}$ & - \\
\hline Tipo de artigo & Aplicação & Aplicação & Aplicação & $\begin{array}{c}\text { Teoria + } \\
\text { Aplicação }\end{array}$ & Aplicação & $\begin{array}{c}\text { Teoria + } \\
\text { Aplicação }\end{array}$ & Aplicação & Aplicação & Aplicação & Aplicação \\
\hline $\begin{array}{l}\text { Outros } \\
\text { modelos }\end{array}$ & - & SFA & SFA & Regressão & - & $\begin{array}{l}\text { SFA; } \\
\text { regressão; } \\
\text { testes } \\
\text { estatísticos }\end{array}$ & - & - & - & - \\
\hline
\end{tabular}

** informações incompletas, por ter sido recuperado sob a forma de resumo. 
Tabela 4: Algumas características das teses e dissertações pesquisadas.

\begin{tabular}{|c|c|c|c|}
\hline Autor & Tema & $\begin{array}{c}\text { Localização } \\
\text { geográfica }\end{array}$ & Tipo de DMU \\
\hline Abreu (2004) & Pecuária de corte & Mato Grosso do Sul & Fazendas/Produtores \\
\hline Aguiar (2003) & Agropecuária & Paraná & Vilas rurais \\
\hline Anjos (2001) & Agropecuária & Rondônia & Assentamentos rurais \\
\hline Barros (2002) & Agricultura & Pernambuco & Empresas agrícolas \\
\hline Brunetta (2004) & Pecuária de leite & Paraná & Fazendas/Produtores \\
\hline Chinelatto Neto (2003) & Agricultura & Minas Gerais & Microrregiões \\
\hline Costa Sobrinho (2006) & Agricultura & Paraná & Municípios \\
\hline Coura (2004) & Agricultura & São Paulo & \\
\hline De Mori (1998) & arroz & Santa Catarina & Fazendas/Produtores \\
\hline Ferreira (2002) & Pecuária de leite & Minas Gerais & Fazendas/Produtores \\
\hline Ferreira (2005) & Pecuária de leite & Brasil (15 estados) & $\begin{array}{c}\text { Cooperativas e } \\
\text { Sociedades de capital }\end{array}$ \\
\hline Gomes (1999) & Pecuária de leite & Minas Gerais & Fazendas/Produtores \\
\hline $\begin{array}{c}\text { Homem de Souza } \\
(2003)\end{array}$ & Pecuária de leite & $\begin{array}{l}\text { (a) Minas Gerais } \\
\text { (b) } 6 \text { estados }\end{array}$ & - \\
\hline Magalhães (2005) & Pecuária de leite & Ceará & Fazendas/Produtores \\
\hline Nogueira (2005) & Agropecuária & Brasil & Microrregiões \\
\hline Pereira (1999) & Agropecuária & Brasil & Estados \\
\hline Roberts (2003) & Pecuária de leite & Rondônia & - \\
\hline Santos (2002) & Agropecuária & Nordeste & Microrregiões \\
\hline Sena (2005) & Agropecuária & Bahia & Municípios \\
\hline Silva (1999) & Agricultura & Pernambuco & Fazendas/Produtores \\
\hline Sousa Júnior (2003) & Aqüicultura & Ceará & Fazendas/Produtores \\
\hline Souza (2002) & Pecuária de leite & Paraná & Fazendas/Produtores \\
\hline Sturion (1996) & Agropecuária & Santa Catarina & Fazendas/Produtores \\
\hline Toresan (1988) & Agricultura & Santa Catarina & Fazendas/Produtores \\
\hline Vilela (2004) & $\begin{array}{l}\text { Cooperativas de } \\
\text { crédito rural }\end{array}$ & São Paulo & $\begin{array}{l}\text { Cooperativas de } \\
\text { crédito rural }\end{array}$ \\
\hline
\end{tabular}

\section{CONSIDERAÇÕES FINAIS}

Este artigo traz uma primeira tentativa de reunir a literatura que trata sobre o uso de DEA em agricultura. Outros autores já realizaram estudos semelhantes para a área agrícola, porém com o enfoque de modelos de fronteiras de produção e eficiência, não sendo específicos para a modelagem DEA. Este artigo é o primeiro que se dedica à revisão do tema 'DEA em agricultura'.

A busca feita foi tão exaustiva quanto possível. Entretanto, acredita-se que ainda existam artigos sobre a temática ENGEVISTA, v. 10, n. 1, p. 27-51, junho 2008 pesquisada, que não estão aqui referenciados. Julga-se que a pesquisa realizada nas bases de dados mais importantes disponíveis foi bastante satisfatória, abrangente, e contemplou uma vasta gama de artigos publicados sobre DEA em agricultura.

Os artigos aqui reunidos mostram a potencialidade de aplicação de DEA na área agrícola, seja na avaliação de desempenho de agricultores/fazendas, cooperativas, centros de pesquisa agropecuária ou de regiões geográficas. Destaque deve ser dado às aplicações a dados nacionais. No entanto, nota-se uma 
carência no que se refere às publicações de casos brasileiros em periódicos de circulação internacional.

A relativa pequena quantidade de artigos publicados em revistas da área de Pesquisa Operacional pode ser explicada pela reduzida quantidade de artigos que fazem uso de modelos teóricos avançados ou que trazem alguma inovação teórica à modelagem DEA. Isto demonstra a potencialidade de inserção para aqueles que desejam dedicar-se a esta temática.

\section{AGRADECIMENTOS}

Ao CNPq, pelo apoio financeiro.

\section{REFERÊNCIAS}

ABAY, C.; MIRAN, B.; GUNDEN, C. An analysis of input use efficiency in tobacco production with respect to sustainability: the case study of Turkey. Journal of Sustainable Agriculture, v. 24, n. 3, p. 123143, 2004.

ABREU, U.G.P. Análise da adoção de tecnologias em sistema extensivo de criação de gado de corte no Pantanal: um estudo de caso. 2004. Tese (Doutorado em Zootecnia), Universidade Federal de Viçosa, Viçosa. 162p.

ABREU, U.G.P.; SANTOS, H.N.; LOPES, P.S.; TORRES, R.A.; BAPTISTA, A.J.M.S. Avaliação da introdução de tecnologias no sistema de produção de gado de corte no Pantanal: análise de eficiência. Revista Brasileira de Zootecnia, v. 36, n. 5, p. 1242-1250, 2006.

AGUIAR, B.C.X.C. Uma metodologia para avaliação da eficiência técnica relativa de vilas rurais utilizando data envelopment analysis. 2003. Dissertação (Mestrado em Métodos Numéricos em Engenharia), Universidade Federal do Paraná, Curitiba. 146p.

ALDAZ, N.; MILLÁN, J.A. Regional productivity of Spanish agriculture in a panel DEA framework. Applied ENGEVISTA, v. 10, n. 1, p. 27-51, junho 2008
Economics Letters, v. 10, n. 2, p. 87-90, 2003.

ALEMDAR, T.; ÖREN, M.N. Measuring technical efficiency of wheat production in southeastern Anatolia with parametric and nonparametric methods. Pakistan Journal of Biological Sciences, v. 9, n. 6, 10881094, 2006.

ALENE, A.D.; MANYONG, V.M.; GOCKOWSK, J. The production efficiency of intercropping annual and perennial crops in southern Ethiopia: a comparison of distance functions and production frontiers. Agricultural Systems, v. 91, p. 51-70, 2006.

ALVAREZ, A.; ARIAS, C. Technical efficiency and farm size: a conditional analysis. Agricultural Economics, v. 30, n. 3, p. 241-250, 2004.

ANJOS, J.C.A. Um sistema de avaliação de produtividade em assentamento rurais utilizando lógica fuzzy. 2001. Dissertação (Mestrado em Ciências da Computação), Universidade Federal de Santa Catarina, Florianópolis. 105p.

ASMILD, M.; HOUGAARD, J.L. Economic versus environmental improvement potentials of Danish pig farms. Agricultural Economics, v. 35, n. 2, p. 171-181, 2006.

AUDIBERT, M.; MATHONNAT, J.; HENRY, M.C. Social and health determinants of the efficiency of cotton farmers in Northern Côte d'Ivoire. Social Science \& Medicine, v. 56, p. 1705-1717, 2003.

BALCOMBE, K.; FRASER, I.; KIM, J.H. Estimating technical efficiency of Australian dairy farms using alternative frontier methodologies. Applied Economics, v. 38, n. 19, p. 2221-2236, 2006.

BALMANN, A.; CZASCH, B. On the efficiency of farms in Brandenburg - a data envelopment analysis. Agrarwirtschaft, v. 50, n. 3, p. 198-203, 2001. 
BANKER, R.D.; CHARNES, A.; COOPER, W.W. Some models for estimating technical scale inefficiencies in Data Envelopment Analysis. Management Science, v. 30, n. 9, p. 1078-1092, 1984.

BAPTISTA, A.J.M.S.; CASTRO, E.R.; TEIXEIRAS, E.C. Discriminação da função de produção, elasticidades de substituição de fatores na agropecuária do Estado de Minas Gerais. Revista de Economia, Agronegócio, v. 2, n. 2, p. 161182, 2004.

BARNES, A.P. Does multi-functionality affect technical efficiency? A nonparametric analysis of the Scottish dairy industry. Journal of Environmental Management, v. 80, p. 287-294, 2006.

BARROS, E.S. A agricultura Irrigada e a Eficiência Técnica das Empresas Agrícolas no Vale do São Francisco: uma comparação do modelo paramétrico de fronteira estocástica com o modelo não paramétrico DEA-V. 2002. Dissertação (Mestrado em Economia), Universidade Federal de Pernambuco, Recife. 86p.

BATTESE, G.E. Frontier production functions and technical efficiency: a survey of empirical applications in agricultural economics. Agricultural Economics, v. 7, n. 1, p. 185-208, 1992.

BAYARSAIHAN, T.; COELLI, T.J. Productivity growth in pre-1990 Mongolian agriculture: spiralling disaster or emerging success? Agricultural Economics, v. 28, p. 121-137, 2003.

BINAM, J.N.; SYLLA, K.; DIARRA, I.; NYAMBI, G. Factors affecting technical efficiency among cofee farmers in Côte d'Ivoire: evidence form the centre west region. R\&D Management, v. 15, n. 1, p. 66-76, 2003.

BINICI, T.; ZULAUF, C.R.; KACIRA, O.O.; KARLI, B. Assessing the efficiency of cotton production on the Harran Plain, Turkey. Outlook on Agriculture, v. 35, n. 3, p. 227-232, 2006.
BOGETOFT, P.; THORSEN, B.J.; STRANGE, N. Efficiency and merger gains in the Danish Forestry Extension Service. Forest Science, v. 49, n. 4, p. 585595, 2003.

BOSETTI, V.; LOCATELLI, G. A data envelopment analysis approach to the assessment of natural parks' economic efficiency and sustainability. The case of Italian national parks. Sustainable Development, v. 14, n. 4, p. 277-286, 2006.

BOZOGLU, M.; CEYHAN, V.; CINEMRE, A.H.; DEMIRYÜREK, K.; KILIC, O. Evaluation of different trout farming systems some policy issues in the Black Sea Region Turkey. Journal of Applied Sciences, v. 6, n. 14, p. 28822888, 2006.

BRAVO-URETA, B.E.; PINHEIRO, A.E. Efficiency analysis of developing country agriculture: a review of the frontier function literature. Agricultural and Resource Economics Review, v. 22, n. 1, p. 1993.

BRAVO-URETA， B.E.; SOLÍS， D.; LÓPEZ, V.H.M.; MARIPANI, J.F.; THIAM, A.; RIVAS, T. Technical efficiency in farming: a meta-regression analysis. Journal of Productivity Analysis, v. 27, n. 1, p. 57-72, 2007.

BRÜMMER, B. Estimating confidence intervals for technical efficiency: the case of private farms in Slovenia. European Review of Agricultural Economics, v. 28, n. 3, p. 285-306, 2001.

BRUNETTA, M.R. Avaliação da eficiência técnica e de produtividade usando analise por envoltória de dados: um estudo de caso aplicado a produtores de leite. 2004. Dissertação (Mestrado em Métodos Numéricos em Engenharia), Universidade Federal do Paraná, Curitiba. $101 \mathrm{p}$.

BYRNES, P.; FÄRE, R.; GROSSKOPF, S.; KRAFT, S., Technical efficiency and size: the case of 1980 Illinois grain farms. 
European Review of Agricultural Economics, v. 14, p. 367-381, 1987.

CHAABAN, J.; RÉQUILLART, V.; TRÉVISIOL, A. The role of technical efficiency in takeovers: evidence from the French cheese industry, 1985-2000. Agribusiness, v. 21, n. 4, p. 545-564, 2005.

CHAMBERS, R.G.; FÄRE, R.; JAENICKE, E. Using dominance in forming bounds on DEA models: the case of experimental agricultural data. Journal of Econometrics, v. 85, n. 1, p. 189-203, 1998.

CHAN, H.L.; CHAN, K.T. The analysis of rural regional disparity in China. Asian Economic Journal, v. 14, n. 1, p. 23-38, 2000.

CHANG, C.C.; HSIEH, T.C. The economic efficiency of the Credit Department of Farmers' Associations in Taiwan. Applied Financial Economics, v. 8, n. 4, p. 409-418, 1998.

CHARNES, A.; COOPER, W.W.; LEWIN, A.Y.; SEIFORD, L.M. Data Envelopment Analysis: Theory, methodology and applications. Boston: Kluwer Academic Publishers, 1994. 513 p.

CHARNES, A.; COOPER, W.W.; RHODES, E. Measuring the efficiency of decision-making units. European Journal of Operational Research, v. 2, p. 429-444, 1978.

CHAVAS, J.; ALIBER, M. An analysis of economic efficiency in agriculture: a nonparametric approach. Journal of Agricultural Economics, v. 18, p. 1-16, 1993.

CHAVAS, J.P.; COX, T.L. A primal-dual approach to nonparametric productivity analysis: The case of U.S. agriculture. Journal of Productivity Analysis, v. 5, n. 4, p. 359-373, 1994.

CHEN, P.C.; YU, M.M.; CHANG, C.C.; HSU, S.H. Productivity change in Taiwan's farmers' credit unions: a nonparametric risk-adjusted Malmquist ENGEVISTA, v. 10, n. 1, p. 27-51, junho 2008 approach. Agricultural Economics, v. 36, n. 2, p. 221-231, 2007.

CHERCHYE, L.; PUYENBROECK, T.V. Profit efficiency analysis under limited information with na application to German farm types. Omega, v. 35, n. 3, p. 335-349, 2007.

CHINELATTO NETO, A. Mudança Tecnológica e uso de fatores de produção na agricultura de Minas Gerais, de 1985 a 1995. 2003. Dissertação (Mestrado em Economia Aplicada), Universidade Federal de Viçosa, Viçosa. 71p.

CHIREMBA, S.; MASTERS, W. The experience of resettled farmers in Zimbabwe. African Studies Quarterly, v. 7, n. 2-3, 2003.

CINEMRE, H.A.; CEYHAN, V.; BOZOĞLU, M.; DEMIRYÜREK, K.; KILIÇ, O. The cost efficiency of trout farms in the Black Sea Region, Turkey. Aquaculture, v. 251, n. 2-4, p. 324-332, 2006.

CLOUTIER, L.M.; ROWLEY, R. Relative technical efficiency: data envelopment analysis and Quebec's dairy farms. Canadian Journal of Agricultural Economics, 41, 169-176, 1993.

COELLI, T. Recent developments in frontier modelling and efficiency measurement, Australian Journal of Agricultural Economics, v. 39, n. 3, p. 219-245, 1995.

COELLI, T.; RAHMAN, S.; THIRTLE, C. Technical, allocative, cost and scale efficiencies in Bangladesh rice cultivation: a non-parametric approach. Journal of Agricultural Economics, v. 53, n. 3,p. 607626, 2002.

COELLI, T.J.; RAO, D.S.P. Total factor productivity growth in agriculture: a Malmquist index analysis of 93 countries, 1980-2000. Agricultural Economics, v. 32, n. 1, p. 115-134, 2005.

COOPER, W.W.; SEIFORD, L.M.; TONE, K. Data Envelopment Analysis: A 
Comprehensive Text with Models, Applications, References and DEA-Solver Software. New York: Kluwer Academic Publishers, 2000. 318p.

COOPER, W.W; SEIFORD, L.M.; ZHU, J. Handbook on data envelopment analysis. Boston: Kluwer Academic Publishers, 2004. 608p.

COSTA SOBRINHO, G.R.C. Estudo nãoparamétrico da eficiência da agricultura do estado do Paraná. 2006. Dissertação (Mestrado em Agronegócios), Fundação Universidade Federal de Mato Grosso do Sul, Cuiabá. 96p.

COURA, R.M. Produtividade total dos fatores na agricultura paulista: 1985-2001. 2004. Dissertação (Mestrado em Economia Aplicada), Universidade Federal de Viçosa, Viçosa. 61p.

DE KOEIJER, T.J.; WOSSINK, G.A.A.; SMIT, A.B.; JANSSENS, S.R.M.; RENKEMA, J.A.; STRUIK, P.C. Assessment of the quality of farmers' environmental management and its effects on resource use efficiency: a Dutch case study. Agricultural Systems, v. 78, p.85103, 2003.

DE KOEIJER, T.J.; WOSSINK, G.A.A.; STRUIK, P.C.; RENKEMA, J.A. Measuring agricultural sustainability in terms of efficiency: the case of Dutch sugar beet growers. Journal of Environmental Management, v. 66, p. 917, 2002.

DE MORI, C. Mensuração da eficiência produtiva de unidades de produção agrícola considerando aspectos agroeconômicos e agroenergéticos. 1998. Dissertação (Mestrado em Engenharia de Produção), Universidade Federal de Santa Catarina, Florianópolis. 103p.

DHARMAPALA, P.S.; ZAIBET, L. Analysis of farmers' efficiency and growth factors in oil exporting Arabian gulf countries: the case of Oman. International Journal of Management and Decision Making, v. 7, n.. 4, p. 377-387, 2006.

ENGEVISTA, v. 10, n. 1, p. 27-51, junho 2008
DHUNGANA, B.R.; NUTHALL, P.L.; NARTEA, G.V. Measuring the economic inefficiency of Nepalese rice farms using data envelopment analysis. The Australian Journal of Agricultural and Resource Economics, v. 48, n. 2, p. 347-369, 2004.

DIAZ-BALTEIRO, L.; HERRUZO, A.C.; MARTINEZ, M.; GONZÁLEZ-PACHÓN, J. An analysis of productive efficiency and innovation activity using DEA: An application to Spain's wood-based industry. Forest Policy and Economics, v. 8, n. 7, p. 762-773, 2006.

DIMARA, E.; PANTZIOS, C.J.; SKURAS, D.; TSEKOURAS, K. The impacts of regulated notions of quality on farm efficiency: A DEA application. European Journal of Operational Research, v. 161, p. 416-431, 2005.

EMROUZNEJAD, A. An extensive bibliography of Data Envelopment Analysis (DEA). Working Papers, Business School, University of Warwick, England, v. I-V, 2001.

FÄRE, R.; GRABOWSKI, R.; GROSSKOPF, S. Technical efficiency of Philippine agriculture. Applied Economics, v. 17, n. 2, p. 205-221, 1985.

FÄRE, R.; GRABOWSKI, R.; GROSSKOPF, S.;KRAFT, S. Efficiency of a fixed but allocatable input: a nonparametric approach. Economics Letters, v. 56, n. 2, p. 187-193, 1997.

FELTHOVEN, R.G. Effects of the american fisheries act on capacity, utilization and technical efficiency. Marine Resource Economics, v. 17, p. 181-205, 2002.

FERNANDEZ-CORNEJO, J. Nonradial technical efficiency and chemical input use in agriculture. Agricultural and Resouce Economics Review, v. 23, n. 1, p. 12-21, 1994.

FERREIRA, A.H. Eficiência de sistemas de produção de leite: uma aplicação da análise envoltório de dados na tomada de 
decisão. 2002. Dissertação (Mestrado em Economia Aplicada), Universidade Federal de Viçosa, Viçosa. 120p.

FERREIRA, M.A.M. Eficiência técnica e de escala de cooperativas e sociedades de capital na indústria de laticínios do Brasil. 2005. Tese (Doutorado em Economia Aplicada), Universidade Federal de Viçosa, Viçosa. 158p.

FERRIER, G.D.; PORTER, P.K. The productive efficiency of US milk processing co-operatives. Journal of Agricultural Economics, v. 42, p.161-173, 1991.

FRASER, I.; CORDINA, D. An application of data envelopment analysis to irrigated dairy farms in Northern Victoria, Australia. Agricultural Systems, v. 59, p. 267-282, 1999.

FRASER, I.; GRAHAM, M. Australasian Efficiency measurement of Australian dairy farms: national and regional performance. Australasian Agribusiness Review, v. 13, 2005.

FRASER, I.; HONE, P. Farm-level efficiency and productivity measurement using panel data: wool production in southwest Victoria. The Australian Journal of Agriculture and Resource Economics, v. 45, n. 2, p. 215-232, 2001.

GALANOPOULOSA,

K.; AGGELOPOULOSB, S.; KAMENIDOUC, I.; MATTAS, K. Assessing the effects of managerial and production practices on the efficiency of commercial pig farming. Agricultural Systems, v. 88, n. 2-3,p. 125-141, 2006.

GARCIA, C.R. Efficiency of selected productive strategies with imprecise data. Acta Horticulturae, v. 659, n. 1, p. 53-60, 2005.

GATTOUFI, S.; ORAL, M.; KUMAR, A.; REISMAN, A. Content analysis of data envelopment analysis literature and its comparison with that of other OR/MS fields. Journal of the Operational Research Society, v. 55, n. 9, p. 911-935, 2004 a.

GATTOUFI, S.; ORAL, M.; KUMAR, A.; REISMAN, A. Epistemology of data envelopment analysis and comparison with other fields of OR/MS for relevance to applications. Socio-Economic Planning Sciences, v. 38, p. 123-140, 2004b.

GILLESPIE, J.; SCHUPP, A.; TAYLOR, G. Factors affecting production efficiency in a new alternative enterprise: the case of the Ratite industry. Journal of Agricultural and Applied Economics, v. 29, n. 2, p. 409-418, 1997.

GOCHT, A.; BALCOMBE, K. Ranking efficiency units in DEA using bootstrapping an applied analysis for Slovenian farm data. Agricultural Economics, v. 35, n. 2, p. 223-229, 2006.

GOMES, A.P. Impactos das transformações da produção de leite sobre o número de produtores e os requerimentos de mão-de-obra e capital. 1999. Tese (Doutorado em Economia Rural), Universidade Federal de Viçosa, Viçosa. $161 \mathrm{p}$.

GOMES, E.G.; MANGABEIRA, J.A.C. Uso de análise de envoltória de dados em agricultura: o caso de Holambra. Engevista, v. 6, n. 1, p. 19-27, 2004.

GOMES, E.G.; MANGABEIRA, J.A.C.; SOARES DE MELLO, J.C.C.B. Análise de envoltória de dados para avaliação de eficiência, caracterização de tipologias em agricultura: um estudo de caso. Revista de Economia, Sociologia Rural, v. 43, n. 4, p. 607-631, 2005.

GOMES, E.G.; SOARES DE MELLO, J.C.C.B.; MANGABEIRA, J.A.C. Fronteira DEA difusa na avaliação de eficiência em agricultura. Investigação Operacional, v. 26, p. 65-88, 2006.

GORTON, M.; DAVIDOVA, S. Farm productivity and efficiency in the CEE applicant countries: a synthesis of results. 
Agricultural Economics, v. 30, n. 1, p. 116, 2004.

GORTON M.; KOVACS, B.; MIZIK, T.; DAVIDOVA, S.; RATINGER, T.; IRAIZOZ, B. An analysis of the performance of commercially oriented farms in Hungary. Post-Communist Economies, v.15, n.3, p.406-416, 2003.

GRAZHDANINOVA, M.; LERMAN, Z. Allocative and technical efficiency of corporate farms in Russia. Comparative Economic Studies, v. 47, p. 200-213, 2005.

GÜL, M. Technical efficiency of apple farming in Turkey: a case study covering Isparta, Karaman and Nigde provinces. Pakistan Journal of Biological Sciences, v. 9, n. 4, p. 601-605, 2006.

HAAG, S.; JASKA, P.V.; SEMPLE, J. Assessing the relative efficiency of agricultural production units in the Blackland Prairie, Texas. Applied Economics, v. 24, p. 559-565, 1992.

HAJI, J. Production efficiency of smallholders' vegetable-dominated mixed farming system in eastern Ethiopia: a nonparametric approach. Journal of African Economies, v. 16, n. 1, P. 1-27, 2007.

HAJI, J.; ANDERSSON, H. Determinants of efficiency of vegetable production in smallholder farms: The case of Ethiopia. Food Economics - Acta Agriculturae Scandinavica, Section C, v. 3, n. 3-4, p. 125-13, 2006.

HANSEN, B.G.; STOKSTAD, G. Measuring financial performance on dairy farms. Food Economics - Acta Agriculturae Scandinavica, Section C, v. 2, n. 2, p. 99-109, 2005.

HELFAND, S.M.; LEVINE, E.S. Farm size and the determinants of productive efficiency in the Brazilian Center-West. Agricultural Economics, v. 31, p. 241-249, 2004.

HOMEM DE SOUZA, D.P. Avaliação de métodos paramétricos e não paramétricos na análise da eficiência da produção de leite. 2003. Tese (Doutorado em Economia Aplicada), Universidade de São Paulo/Escola Superior de Agricultura Luiz de Queiroz, Piracicaba. 136p.

HOMEM DE SOUZA, D.P.; ALVES, E. Dois critérios para avaliação de eficiência técnica. Revista de Economia, Agronegócio, v. 1, n. 3, p. 373-396, 2003.

HORIE, T.; YAMAGUCHI, M. Productivity growth, efficiency change and technical change in Japanese agriculture: 1965-1995. Japanese Journal of Rural Economics, v. 8, p. 64-78, 2006.

JAENICKE, E.C. Testing for intermediate outputs in dynamic DEA models: accounting for soil capital in rotational crop production and productivity measures. Journal of Productivity Analysis, v. 14, p. 247-266, 2000.

JAFORULLAH, M.; WHITEMAN, J. Scale efficiency in the New Zealand dairy industry: a nonparametric approach. The Australian Journal of Agricultural and Resource Economics, v. 43, n. 4, p. 523$541,1999$.

JHA, R.; CHITKARA, P.; GUPTA, S. Productivity, technical and allocative efficiency and farm size in wheat farming in India: a DEA approach. Applied Economics Letters, v. 7, p. 1-5, 2000.

KALIBA, A.R.; ENGLE, C.R.; DORMAN, L. Efficiency change and technological progress in the U.S. catfishprocessing sector, 1986 to 2005. Aquaculture Economics \& Management, v. 11, n. 1, pages 53-72, 2007.

KALIBA, A.R.M. Technical efficiency of smallholder dairy farms in central Tanzania. Quarterly Journal of International Agriculture, v. 43, n. 1, p. 3955, 2004.

KAMRUZZAMAN, M.; MANOS, B.; BEGUM, M.A.A. Evaluation of economic efficiency of wheat farms in a region of Bangladesh under the input orientation 
model. Journal of the Asia Pacific Economy, v. 11, n. 1, p. 123-142, 2006.

$\mathrm{KAO}, \mathrm{C}$.. Data envelopment analysis in measuring the efficiency of forest management. Journal of Environmental Management, v. 38, p. 73-83, 1993.

KIM, J.M. Efficiency analysis of sustainable and conventional farms in the $r$ public of Korea with special reference to the Data Envelopment Analysis (DEA). Journal of Sustainable Agriculture, v. 18, n. 4, p. 9-26, 2001.

KLEINHANSS, W.; MURILLO, C.; SAN JUAN, C.; SPERLICH, S. Efficiency, subsidies, and environmental adaptation of animal farming under CAP. Agricultural Economics, v. 36, n. 1, p. 49-65, 2007.

KRASACHAT, W. Technical efficiencies of rice farms in Thailand: a non-parametric approach. Journal of American Academy of Business, v. 4, n. 1/2; p. 64-69, 2004.

KUOSMANEN, T.; POST, T.; SIPILÄINEN, T. Shadow price approach to total factor productivity measurement: with an application to Finnish grass-silage production. Journal of Productivity Analysis, v. 22, p. 95-121, 2004.

KWON, O.S.; LEE, H. Productivity improvement in Korean rice farming: parametric and non-parametric analysis. The Australian Journal of Agricultural and Resource Economics, v. 48, n. 2, p. 323346, 2004.

LANSINK, A.O.; BEZLEPKIN, I. Productivity growth and inter-sector spillover in Dutch horticulture, 1976-1995 Agricultural Economics, v. 34, n. 1, p. 109-116, 2006.

LANSINK, A.O; PIETOLA, K.; BÄCKMAN, S. Effciency and productivity of conventional and organic farms in Finland 1994-1997. European Review of Agricultural Economics, v. 29, n. 1, p. 5165, 2002.

LANSINK, A.O.; REINHARD, S. Investigating technical efficiency and ENGEVISTA, v. 10, n. 1, p. 27-51, junho 2008 potential technological change in Dutch pig farming. Agricultural Systems, v. 79, p. 353-367, 2004.

LANSINK, A.O.; SILVA, E. Nonparametric production analysis of pesticides use in the Netherlands. Journal of Productivity Analysis, v. 21, p. 49-65, 2004.

LATRUFFE, L.; BALCOMBE， K.; DAVIDOVA, S.; ZAWALINSKA, K. Determinants of technical efficiency of crop and livestock farms in Poland. Applied Economics, v. 36, n. 12, p. 125563, 2004.

LEE, J.Y. Using DEA to measure efficiency in forest and paper companies. Forest Products Journal, v. 55, n. 1, p. 5866, 2005.

LIN, F.J.; PENG, K.C.; CHENG, M.Y. A study on comparison and improvement of management efficiency in agriculture cooperatives. Journal of the Agricultural Association of China, v. 5, n. 2, p. 103128, 2004.

LISSITSA, A.; ODENING, M. Efficiency and total factor productivity in Ukrainian agriculture in transition. Agricultural Economics, v. 32, n. 3, p. 311-325, 2005.

LOVELL, C.A.K. Future research opportunities in efficiency and productivity analysis. Permanent Seminar on Efficiency and Productivity - Efficiency Series Paper 1/2001, Departamento de Economía, Universidad de Oviedo, 18 p., 2001.

LUND, M.; JACOBSEN, B.H.; HANSEN, L.C.E. Reducing non-allocative costs on Danish dairy farms: Application of nonparametric methods. European Review of Agricultural Economics, v. 20, p. 327-341, 1993.

LUTFI, M.; OIDA, A.; NAKASHIMA, H.; OHDOI, K. Evaluation of small mechanized farms in east Java by data envelopment analysi. International Agricultural Engineering Journal, v. 15, n. 4, p. 189-196, 2006. 
MAGALHÃES, K.A. Análise da Eficiência técnica e do perfil socioeconômico dos produtores de leite do município de Sobral - Ceará. 2005. (Mestrado em Economia Rural), Universidade Federal do Ceará, Fortaleza. $111 \mathrm{p}$.

MALANA, N.M.; MALANO, H.M. Benchmarking productive efficiency of selected wheat areas in Pakistan and India using data envelopment analysis. Irrigation and Drainage, v. 55, n. 4, p. 383-394, 2006.

MANAGI, S.; KAREMERA, D. Input and output biased technological change in US agriculture. Applied Economics Letters, v. 11, p. 283-286, 2004.

MANOS, B.; PSYCHOUDAKIS, A. Investigation of the relative efficiency of dairy farms using data envelopment analysis. Quarterly Journal of International Agriculture, v. 36, n. 2, p. 188-197, 1997.

MAO, W.; KOO, W.W. Productivity growth, technological progress, and efficiency change in Chinese agriculture after rural economic reforms: a DEA approach. China Economic Review, v. 8, n. 2, p. 157-174, 1997.

MATHIJS, E.; BLAAS, G.; DOUCHA, T. Organisational form and technical efficiency of Czech and Slovak farms. MOCT-MOST, v. 9, p. 331-344, 1999.

MBAGA, M.D.; ROMAIN, R.; LARUE, B. LEBEL, L. Assessing technical efficiency of Québec dairy farms. Canadian Journal of Agricultural Economics, v. 51, n. 1, p. 121-137, 2003.

MOSHEIM, R. Organizational type and efficiency in the Costa Rica coffee processing sector. Journal of Comparative Economics, v. 30, p. 296-316, 2002.

NGHIEM, H.S.; COELLI, T. The effect of incentive reforms upon productivity: evidence from the Vietnamese rice industry. The Journal of Development Studies, v. 39, n. 1, p.74-93, 2002.
NKAMLEU, G.B. Productivity growth, technical progress and efficiency change in African agriculture. African Development Bank 2004, p. 203-222, 2004,

NOGUEIRA, M.A. Eficiência técnica na agropecuária das microrregiões brasileiras. 2005. Tese (Doutorado em Economia Aplicada), Universidade Federal de Viçosa, Viçosa, 105p.

ÖREN, M.N.; ALEMDAR, T. Technical efficiency analysis of tobacco farming in southeastern Anatolia. Turkish Journal of Agriculture and Forestry, v. 30, p. 165172, 2006.

OSBORNE, S.; TRUEBLOOD, M.A. An examination of economic efficiency of Russian crop production in the reform period. Agricultural Economics, v. 34, n. 1, p. 25-38, 2006.

OTSUKI, T.; HARDIE, I.W.; REIS, E.J. The implication of property rights for joint agriculture-timber productivity in the Brazilian Amazon. Environment and Development Economics, v. 7, p. 299-323, 2002.

PASCOE, S.; HERRERO, I. Estimation of a composite fish stock index using data envelopment analysis. Fisheries Research, v. 69, p. 91-105, 2004.

PAUL, C.M; NEHRING, R.; BANKER, D. Scale economies and efficiency in U.S. agriculture: are traditional farms history? Journal of Productivity Analysis, v. 22, p. 185-205, 2004.

PENG, K.C. An evaluating on the relative management efficiency of agricultural cooperatives in Taiwan. Journal of the Agricultural Association of China, v. 4, n. 2, p. 156-175, 2003.

PEREIRA, M.F. Evolução da fronteira tecnológica múltipla e da produtividade total dos fatores do setor agropecuário brasileiro e 1970 a 1996. 1999. Tese (Doutorado em Engenharia de Produção), Universidade Federal de Santa Catarina, Florianópolis. 142p. 
PEREIRA, M.F.; SILVEIRA, J.S.T.; LANZER, E.A.; SAMOHYL, R.W. Productivity growth and technological progress in the Brazilian agricultural sector. Pesquisa Operacional, v. 22, n. 2, p.133-146, 2002.

PHILLIPS, F. 25 years of data envelopment analysis. International Journal of Information Technology \& Decision Making, v. 4, n. 3, p. 317-323, 2005.

PICAZO-TADEO, A.J.; REIGMARTÍNEZ, E. Agricultural externalities and environmental regulation: evaluating good practice in citrus production. Applied Economics, v. 38, n. 11, p. 1327-1334, 2006a.

\section{PICAZO-TADEO, A.J.; REIG- MARTÍNEZ, E. Farmers' costs of environmental regulation: reducing the consumption of nitrogen in citrus farming. Economic Modelling, v. 24, p. 312-328, 2007.}

PICAZO-TADEO, A.J.; REIGMARTINEZ, E. Outsourcing and efficiency: the case of Spanish citrus farming. Agricultural Economics, v. 35, n. 2, p. 213-222, 2006b.

PIESSE, J.; DOYER, T.; THIRTLE, C.; VINK, N. The changing role of grain cooperatives in the transition to competitive markets in South Africa. Journal of Comparative Economics, v. 33, p. 197-218, 2005.

PIESSE, J.; VON BACH, H.S.; THIRTLE, C.; VAN ZYL, J. The efficiency of smallholder agriculture in South Africa. Journal of International Development, v. 8, n. 1, p. 125-144, 1996.

PIOT-LEPETIT, I. AND VERMERSCH, D. Pricing organic nitrogen under the weak disposability assumption: an application to the French pig sector. Journal of Agricultural Economics, v. 49, p. 85-99, 1998.
PIOT-LEPETIT, I.; RAINELLI, P. Breathing room in farm management as determined by inefficiency measurements. INRA Productions Animales, v. 9, n. 5, p. 367-377, 1996.

PIOT-LEPETIT, I.; VERMERSCH，D.; WEAVER, R.D. Agriculture's environmental externalities: DEA evidence for French agriculture. Applied Economics, v. 29, p. 331-338, 1997.

PSYCHOUDAKIS, A.; DIMITRIADOU, E. An application of data envelopment analysis in a sample of dairy farms. Medit, v. 10, n. 3, p. 46-50, 1999.

RAO, D.S.P.; COELLI, T.J. Catch-up and convergence in global agricultural productivity. Indian Economic Review, v. 39, n. 1, p. 123-148, 2004.

RAY, S. Measurement and test of efficiency of farms in linear programming models: a study of West Bengal farms. Oxford Bulletin of Economics and Statistics, v. 47, n. 4, p. 371-386, 1985.

REIG-MARTÍNEZ, E.; PICAZO-TADEO, A.J. Analysing farming systems with Data Envelopment Analysis: citrus farming in Spain. Agricultural Systems, v. 82, p. 1730, 2004.

REINHARD, S.; KNOX LOVELL, C.A.; THIJSSEN, G.J. Environmental efficiency with multiple environmentally detrimental variables; estimated with SFA and DEA. European Journal of Operational Research, v. 121, p. 287-303, 2000.

ROBERTS, D.B. Eficiência da pequena produção de leite no estado de Rondônia. 2003. Dissertação (Mestrado em Economia Aplicada), Universidade Federal de Viçosa, Viçosa. 84p.

RODRIGUEZ-DIAZ, J.A.; CAMACHOPOYATO, E.; LOPEZ-LUQUE, R. Application of data envelopment analysis to studies of irrigation efficiency in andalusia. Journal of Irrigation and Drainage Engineering, v. 130, n. 3; p. 175183, 2004a. 
RODRIGUEZ-DIAZ, J.A.; POYATO, E.C.; LUQUE, R.L. Applying benchmarking and data envelopment analysis (DEA) techniques to irrigation districts in Spain. Irrigation and Drainage, v. 53, p. 135-143, 2004b.

RUSIELIK, R.; SWITYK, M. The DEA method application in efficiency measurement of agriculture in Poland in 1990 and 1995. Folia Universitatis Agriculturae Stetinensis, Oeconomica, v. 36, p. 179-190, 1999.

SANTOS, F.A.A. Condicionantes da eficiência na agropecuária do Nordeste. 2002. Tese (Doutorado em Economia Aplicada), Universidade Federal de Viçosa, Viçosa. 148p.

SANTOS, J.A.; VIEIRA, W.C.; BAPTISTA, A.J.M.S. Eficiência técnica na produção de leite em pequenas propriedades da microrregião de ViçosaMG. Revista de Economia, Agronegócio, v. 2, n. 2, p. 261-290, 2004.

SARKER, D.; DE, S. Non-parametric approach to the study of farm efficiency in agriculture. Journal of Contemporary Asia, v. 34 n. 2, p. 207-220, 2004.

SARRIS, A.H.; DUCHA, T.; MATHIJS, E. Agricultural restructuring and in central and eastern Europe: implications for competitiveness and rural development. European Journal of Agricultural Economics, v. 26, n. 3, p. 305-329, 1999.

SAUER, J.; ABDALLAH, J.M. Forest diversity, tobacco production and resource management in Tanzania. Forest Policy and Economics, v. 9, n. 5, p. 421-439, 2007.

SEDIK, D.; TRUEBLOOD, M.; ARNADE, C. Corporate farm performance in Russia, 1991-1995: an efficiency analysis. Journal of Comparative Economics, v. 27, n. 3, p. 514-533, 1999.

SEIFORD, L.M. A bibliography for Data Envelopment Analysis (1978-1996).
Annals of Operations Research, v. 73, p. 393-438, 1997.

SENA, N.A.M.O. Fronteira tecnológica, alocação de fatores e eficiência na agropecuária da microrregião ItabunaIlhéus, estado da Bahia. 2005. Dissertação (Mestrado em Ciências Agrárias), Universidade Federal da Bahia, Salvador. $127 \mathrm{p}$.

SENA, N.A.M.O; PEREIRA FILHO, C.A. Medidas de eficiência técnica, de escala na agropecuária da microrregião Itabuna-Ilhéus, estado da bahia, aplicando a análise envoltória de dados. Artigocientífico, v. 5, p. 1292-1318, 2006.

SHAFIQ, M.; REHMAN, T. The extent of resource use inefficiencies in cotton production in Pakistan's Punjab: an application of data envelopment analysis. Agricultural Economics, v. 22, p. 321-330, 2000.

SHARMA, K.R.; LEUNG, P.; ZALESKI , H.M. Productive efficiency of the swine industry in Hawaii: stochastic frontier vs. data envelopment analysis. Journal of Productivity Analysis, v. 8, p. 447-459, 1997.

SHARMA, K.R.; LEUNG, P.S. A review of production frontier analysis for aquaculture management. Aquaculture Economics \& Management, v. 7, n. 1-2, p. 15-34, 2003.

SHARMA, K.R.; LEUNG, P.S.; CHEN, H.; PETERSON, A. economic efficiency and optimum stocking densities in fish polyculture: an application of data envelopment analysis to Chinese fish farms. Aquaculture, v. 180, p. 207-221, 1999a.

SHARMA, K.R.; LEUNG, P.S.; ZALESKI, H.M. Technical, allocative and economic efficiencies in swine production in Hawaii: a comparison of parametric and nonparametric approaches. Agricultural Economics, v. 20, p. 23-35, 1999b. 
SILVA, J.L.M. A eficiência dos colonos na agricultura irrigada no Vale de Aso Francisco: uma analise comparativa de modelos de fronteira paramétrica e não paramétrica. 1999. Tese (Doutorado em Economia), Universidade Federal de Pernambuco, Recife. 142p.

SILVA, J.L.M.; SAMPAIO, Y.S.B. A eficiência técnica dos colonos nos perímetros irrigados em Petrolina, Juazeiro: uma análise de modelos de fronteiras de produção. Revista Econômica do Nordeste, v. 33, n. 2, p. 159-179, 2002.

SINGH, S.; COELLI, T.J.; FLEMING, E. Performance of dairy plants in the cooperative and private sectors in India. Annals of Public and Cooperative Economics, v. 72, n. 4, p. 453-479, 2001.

SOMWARU, A.; NEHRING, R. A graph efficiency multiproduct model of corn/livestock farming: accounting for nitrate pollution. Annals of Operations Research, v. 68, p. 379-408, 1996.

SOUSA JÚNIOR, J.P. Análise da eficiência da produção de camarão marinho em cativeiro no estado do Ceará. 2003. Dissertação (Mestrado em Economia Rural), Universidade Federal do Ceará, Fortaleza. 107p.

SOUSA JÚNIOR, J.P.; KHAN, A.S.; LIMA， P.V.P.S.; MADRID, R.M.M. Produção de camarão marinho em cativeiro: uma análise de eficiência técnica, alocativa, de custos. Revista de Economia Aplicada, v. 9, n. 2, p. 205-224, 2005.

SOUZA, G.S. Significância de efeitos técnicos na eficiência de produção da pesquisa agropecuária. Revista Brasileira de Economia, v. 60, n. 1, p. 69-86, 2006.

SOUZA, G.S.; ÁVILA, A.F.D.; ALVES, E. Technical efficiency of production in agricultural research. Scientometrics, v. 46, n. 1, p. 141-160, 1999.

SOUZA, G.S.; GOMES, E.G.; MAGALHÃES, M.C.; ÁVILA, A.F.D.
Economic efficiency of Embrapa $s$ research centers and the influence of contextual variables. Pesquisa Operacional, 2007.

SOUZA, P.C.T. Uma metodologia baseada em DEA para avaliação da eficiência técnica de produtores de leite. 2002. Dissertação (Mestrado em Métodos Numéricos em Engenharia), Universidade Federal do Paraná, Curitiba. 75p.

SOWLATI, T. Efficiency studies in forestry using data envelopment analysis. Forest Products Journal, v. 55, n. 1, p. 4957, 2005.

STOKES, J.R.; TOZER, P.R.; HYDE, J. Identifying efficient dairy producers using data envelopment analysis. Journal of Dairy Science, v. 90 , n. 5, p. 2555-2562, 2007.

STURION, L. Avaliação do potencial de analise de envoltória de dados do diagnóstico da produtividade de unidades de produção agropecuária. 1996. Dissertação (Mestrado em Engenharia de Produção), Universidade Federal de Santa Catarina, Florianópolis. 140p.

SUEYOSHI, T. DEA non-parametric ranking test and index measurement: slackadjusted DEA and an application to Japanese agriculture cooperatives. Omega, v. 27 , p. $315-326,1999$.

SUEYOSHI, T.; HASEBE, T.; ITO, F.; SAKAY, J.; OSAWA, W. DEA-bilateral performance comparison: an application to Japan agricultural co-operatives (Nokyo). Omega, v. 26, n. 2, p. 233-248, 1998.

TAVARES, G. A bibliography of Data Envelopment Analysis (1978-2001). Rutcor Research Report RRR 01-02, Rutgers Center for Operations Research, USA, 186 p., 2002.

THIAM, A.; BRAVO-URETA, B.E.; RIVAS, T.E. Technical efficiency in developing country agriculture:a metaanalysis. Agricultural Economics, v. 25, p. 235-243, 2001. 
THIELE, H.; BRODERSEN, C.M. Differences in farm efficiency in market and transition economies: empirical evidence from West and East Germany. European Review of Agricultural Economics, v. 26, n. 3, p. 331-347, 1999.

THOMPSON, R.G.; LANGEMEIER, L.N.; LEE, C.T.; LEE, E.; THRALL, R.M. The role of multiplier bounds in efficiency analysis with application to Kansas farming. Journal of Econometrics, v. 46, n. 1-2, p. 93-108, 1990.

TIMMER, M.P.; LOS, B. Localized innovation and productivity growth in Asia: an intertemporal DEA approach. Journal of Productivity Analysis, v. 23, 4764, 2005.

TINGLEY, D.; PASCOE, S. Factors affecting capacity utilisation in English Channel fisheries. Journal of Agricultural Economics, v. 56, n. 2, p. 287-305, 2005.

TIPI, T.; REHBER, E. Measuring technical efficiency and total factor productivity in agriculture: the case of the South Marmara region of Turkey. New Zealand Journal of Agricultural Research, v. 49, n. 2, p. 137-145, 2006.

TORESAN, L. Sustentabilidade e desempenho produtivo na agricultura: uma abordagem multidimensional aplicada a empresas agrícolas. 1998. Tese (Doutorado em Engenharia de Produção), Universidade Federal de Santa Catarina, Florianópolis. 133p.

TOWNSEND, R.F.; KIRSTEN, J.; VINK, N. Farm size, productivity and returns to scale in agriculture revisited: a case study of wine producers in South Africa. Agricultural Economics, v. 19, n. 1,p. 175180, 1998.

TUPY, O. ; YAMAGUCHI, L.C.T. Identificando benchmarks na produção de leite. Revista de Economia, Sociologia Rural, v. 40, n. 1, p. 81-96, 2002.

TUPY, O.; ESTEVES, S.N.; VIEIRA, M.C. Eficiência produtiva de cooperativas

ENGEVISTA, v. 10, n. 1, p. 27-51, junho 2008 de laticínios do estado de São Paulo. Informações Econômicas, v. 33, n. 7, p. 38-46, 2003a.

TUPY, O.; FREITAS,A.R.; ESTEVES, S.N.; SCHIFFLER, E.A.; VIEIRA, M.C. Eficiência econômica na produção de leite tipo B no estado de São Paulo. Informações Econômicas, v. 33, n. 2, p. 14-20, $2003 b$.

TURK, J. Productive performance of Slovenian milk farms under the former regime and during the first stages of economic system transformation. Eastern European Economics, v. 36, n. 2, p. 7-31, 1998.

VARGA, T. Potential for efficiency improvement of Hungarian agriculture. Studies in Agricultural Economics Budapest, v. 104, p. 85-107, 2006.

VENNESLAND, B. Measuring rural economic development in Norway using data envelopment analysis. Forest Policy and Economics, v. 7, p. 109-119, 2005.

VICENTE, J.R. Mudança tecnológica, eficiência, produtividade total de fatores na agricultura brasileira, 1970-95. Economia Aplicada, v. 8, n. 4, p. 729-760, 2004.

VILELA, D.L. Utilização do método analise envoltória de dados para avaliação do desempenho econômico de cooperativas de crédito. 2004. Dissertação (Mestrado em Engenharia de Produção), Universidade de São Paulo, São Carlos. $144 \mathrm{p}$.

WADUD, A.; WHITE, B. Farm household efficiency in Bangladesh: a comparison of stochastic frontier and DEA methods. Applied Economics, v. 32, p. 1665-1673, 2000.

WHITTAKER, G. The relation of farm size and government programme benefits: na application of data envelopment analysis to policy evaluation. Applied Economics, v. 26, p. 469-478, 1994.

WOSSINK, A.; DENAUX, Z.S. Environmental and cost efficiency of 
pesticide use in transgenic and conventional cotton production. Agricultural Systems, v. 90, p. 312-328, 2006.

YANG, Y.; MA, C.; ZHANG, D. Data envelopment. Analysis and its application in the benefit analysis of milk cow production. Transactions of the Chinese Society of Agricultural Engineering, v. 10, n. 1, p. 16, 1994.

ZAIBET, L.; DHARMAPALA, P.S. Efficiency of government-supported horticulture: the case of Oman. Agricultural Systems, v. 62, p. 159-168, 1999.

ZAIBET, L.; DHARMAPALA, P.S.; BOUGHANMI, H.; MAHGOUB, O.; AL-
MARSHUDI, A. Social changes, economic performance and development: the case of goat production in Oman. Small Ruminant Research, v. 54, p. 131-140, 2004.

ZOFIO, J.L.; LOVELL, C.A.K. Graph effciency and productivity measures: an application to US agriculture. Applied Economics, v. 33, p. 1433-1442, 2001.

ZONG, X. Approach to estimating the agricultural mechanization contribution rate by two models in the DEA method. Transactions of the Chinese Society of Agricultural Engineering, v. 22, n. 5, p. 2023, 2006. 